\title{
Competencias socioafectivas de profesores de escuelas secundarias públicas
}

\section{Socio-affective skills of Public Middle School Teachers of Chihuahua}

\author{
Miguel Antonio Aguirre Medrano ${ }^{1}$
}

\begin{abstract}
Resumen
La educación es un proceso en el cual convergen las dimensiones cognitiva, biológica y socioemocional del individuo. Esta última es de especial importancia pues repercute en diversos ámbitos como la convivencia escolar, el ambiente de aprendizaje y el rendimiento académico. Aunque la educación integral -la cual incluye varios aspectos, entre ellos el emocionalse ha abordado en las leyes y normativas de nuestro país, en la práctica no se ha logrado atender a cabalidad. Por lo anterior cobra relevancia identificar y describir cuál es la percepción de la emocionalidad desde la óptica de estudiantes y profesores de escuelas secundarias públicas de la ciudad de Chihuahua, para la cual se diseñaron dos instrumentos que recuperan diversas categorías relacionadas con este ámbito, mediante escala tipo Likert. En esta ocasión se muestran hallazgos parciales de la categoría Competencias Socioafectivas Docentes. El enfoque de la investigación es cuantitativo, su alcance es descriptivo y el tipo de estudio es transversal. La aplicación de instrumentos se llevó a cabo durante el semestre enero-junio de 2018 y se eligió una muestra intencional de 398 estudiantes en escuelas secundarias federales y estatales, así como sus diversas modalidades -telesecundaria, general y técnica, pertenecientes a los tres grados y ambos turnos. Los hallazgos dan cuenta de profesores que, en su mayoría, atienden los ámbitos cognitivos y socioafectivos de los estudiantes que día tras día reciben en sus aulas.
\end{abstract}

\footnotetext{
1 Miguel Antonio Aguirre Medrano. Docente frente en licenciatura y posgrado en la Escuela Normal Superior José E. Medrano R., Universidad del Valle de México campus Chihuahua y en la Universidad Regional del Norte, campus Chihuahua, México. Correo electrónico: m.aguirremedrano@gmail.com

ID https://orcid.org/0000-0002-5741-5018
} 
RECIE. Revista Electrónica Científica de Investigación Educativa Vol. 4, núm. 2, enero-diciembre 2019, pp. 1179-1187.

\title{
Palabras clave
}

Competencias emocionales, competencias docentes, dinámica de grupo, ambientes de aprendizaje.

\begin{abstract}
Education is a process in which the cognitive, biological and socio-emotional dimensions of the individual converge. This last one it's especially important due to it has repercussions in various areas such as school coexistence, the learning environment and academic performance. Although comprehensive education - which includes several aspects, including emotional - has been addressed in the laws and regulations of our country, in practice it has not been fully met. Therefore, it is important to identify and describe the perception of emotionality from the perspective of students and teachers of public middle schools in the city of Chihuahua for which two instruments were designed that include various categories related to this topic, using a Likert-type scale. On this case, partial findings of the Socioafective Teaching Competencies category are shown. The focus of the research is quantitative, its scope is descriptive and the type of study is cross-sectional. The application of instruments was carried out during the January-June semester of 2018 and an intentional sample of 398 students was chosen in federal and state secondary schools as well as its diverse modalities telesecundaria, general and technical. The findings show teachers who, for the most part, attend to the cognitive and socio-affective scopes of the students they receive day after day in their classrooms.
\end{abstract}

\section{Keywords}

Socio-emotional competencies, teaching competences, group dynamic, learning environments.

\section{Introducción}

La educación es un proceso integral que atiende la formación cognitiva, social y emocional de los estudiantes, su carácter holístico permite que el ser humano se reconozca como un sujeto único pero al mismo tiempo que forma parte de una sociedad en la cual existen reglas, normas, leyes y prácticas sociales que atender. En palabras de Casassus:

...una escuela es fundamentalmente una comunidad de relaciones y de interacciones orientadas al aprendizaje, donde el aprendizaje depende principalmente del tipo de relaciones que se establezcan en la escuela y en el aula (2009, pág. 239). 
Y añade que este clima emocional es el resultado de tres variables: el tipo de vínculo entre profesor y alumnos, el vínculo entre estudiantes y el clima resultante de las primeras dos variables.

La formación básica en los sistemas educativos de los diversos países ha obedecido a las necesidades apremiantes de su contexto espaciotemporal, esto es, a lo largo de la historia, los sistemas educativos nacionales han transitado desde modelos con un enfoque situado en promover el nacionalismo, capacitar para el trabajo, promover la cognición del individuo y la resolución de problemas hasta la promoción de un enfoque más humanista (Braslavsky, 1999). De este modo, "los cambios económicos, sociales y de otra índole que afectaron a la sociedad humana en los últimos años han obligado a reconsiderar los conocimientos, las aptitudes y los valores requeridos para llevar una vida satisfactoria" (Organización de las Naciones Unidas para la Educación, la Ciencia y la Cultura -UNESCO, 2000, pág. 19).

Lo anterior ha devenido en una exigencia cada vez mayor en que la educación impartida en los centros escolares atienda la multidimensionalidad del individuo, es decir, su mente, su cuerpo y sus emociones. Desde principios de 1990 -en la Declaración Mundial de la Educación para Todos (UNESCO, 1994) y posteriormente en el informe Delors (1996)- se ha insistido en que la educación debe atender diversas dimensiones o dominios como son el saber saber, el saber hacer, el saber ser y el saber convivir, pues se observó que era necesario complementar la formación del individuo en todas sus esferas y no sólo en lo cognitivo.

En México se ha expresado esta inquietud en diversas normativas, como son el artículo tercero constitucional, el Plan Nacional de Desarrollo y el Programa Sectorial de Educación, no obstante, la disyuntiva se presenta más allá del discurso, en el aula, en el centro escolar, en la cotidianidad, pues pareciera que la omisión de las emociones es la moneda de uso común en las escuelas de nuestro país.

Así, puede apreciarse que se ha optado por un modelo antiemocional que mediante la coerción, la disciplina y el orden ha generado condiciones de aprendizaje que no promueven el desarrollo de la multidimensionalidad del individuo que se forma en las aulas y que devienen en índices de violencia, deserción y prácticas disruptivas en las aulas (Ducoing y Barrón, 2017; Moreno, 2010; Casassus, 2009; Reimers, 2001).

En este tenor, es posible señalar que la escuela secundaria es un nivel educativo complejo, en el cual convergen situaciones académicas que recuperan logros y áreas de oportunidad de preescolar y primaria; aspectos psicosociales y biológicos propios de los adolescentes y que inciden en la 
dinámica áulica por su misma naturaleza, la formación docente de directivos y profesorado, el manejo de la disciplina y prevención de la violencia por parte de las autoridades escolares y los docentes así como un enfoque formativo que, en muchos de los casos, enfatiza más los castigos que la formación. Asimismo, estos aspectos se ven reflejados en las emociones de los estudiantes, en la manera cómo perciben la interacción con sus pares y con sus profesores, pues es con ellos con quienes pasa una cantidad importante de horas diariamente. Esta emocionalidad en muchas ocasiones ha sido invisibilizada y con ello se ha perdido la oportunidad de comprenderla y de incidir en ella.

Por lo anterior, se ha iniciado un estudio que indaga acerca de la percepción de la emocionalidad en las escuelas secundarias desde la óptica de profesores y estudiantes, los mecanismos más utilizados para propiciar orden y disciplina, las emociones más recurrentes en el aula, la interacción docente-alumno, las competencias socioafectivas docentes más desarrolladas y el estilo de comunicación del profesorado. En este tenor, se abordarán resultados parciales de la investigación, centrándose únicamente en los hallazgos de la dimensión competencias socioafectivas docentes -la empatía, la inmediatez, el respeto y el enfoque de enseñanza- que manifiestan los docentes. El objetivo será describir las competencias socioafectivas de los profesores de las escuelas secundarias públicas de la ciudad de Chihuahua, desde la perspectiva del alumnado.

En primer lugar, es conveniente definir a las competencias emocionales como "el conjunto de conocimientos, capacidades, habilidades $\mathrm{y}$ actitudes necesarias para tener conciencia, comprender, expresar y regular de forma apropiada a los fenómenos emocionales" (Bisquerra, 2009 citado en Espinosa, 2013, pág. 37). Sin embargo, la formación docente no contempla en sus planes de estudio el desarrollo de competencias docentes que atiendan la socioemocionalidad -ni la propia ni la de sus estudiantestal como señala Toro, "darse cuenta de las emociones sigue siendo esa asignatura pendiente en el currículum formativo de la mayor parte de los educadores, una laguna inmensa en la que naufraga todo un sistema educativo que no las atiende y las obvia [...]" (2005, pág. 179).

En segundo término, y ante la carencia de una preparación pertinente, se han realizado esfuerzos para detectar las competencias socioafectivas docentes que se requieren para llevar a cabo una enseñanza que atienda la emocionalidad del estudiante. Al respecto, se ha considerado la propuesta de Olson y Wyett (2000) quienes indican que un docente efectivo se apoya en la autenticidad -definida como la capacidad para 
mostrarse genuino con sus estudiantes-, el respeto -la capacidad de percibir la diversidad, valorarla y reconocer en otros la legitimidad de ser único- y la empatía -procurar en todo momento la comprensión de las razones, motivos o circunstancias que inciden para que un individuo actúe de tal o cual forma así como el abordaje que al respecto realiza Shulman (citado en García, 2009) al referir que la enseñanza puede enfocarse en aspectos cognitivos -conocimientos, fórmulas, teorías...- o afectivos -interés por el ser humano que está formando.

\section{Metodología}

Para el presente estudio, se ha optado por el enfoque cuantitativo, la investigación será descriptiva, pues "se pretende medir o recoger información de manera independiente o conjunta sobre los conceptos o las variables a las que se refieren, esto es, su objetivo no es indicar cómo se relacionan estas" (Fernández y Baptista, 2010, p. 122). El tipo de estudio será transversal, pues se llevará a cabo "en una situación y población concreta en un momento determinado" (Martínez, 2007, p. 16).

En la búsqueda de una población representativa se eligió una muestra intencional que reflejara los subsistemas y las modalidades que existen en la escuela secundaria en el municipio de Chihuahua, esto es, subsistema estatal y federal así como las modalidades de telesecundaria, técnicas y generales.

Asimismo, se diseñó un instrumento que consta de siete apartados, entre los cuales se retoma el correspondiente a las competencias socioafectivas docentes, el cual fue pilotado en estudiantes con características similares a los de la población de estudio y validado por expertos (psicólogos clínicos que además ejercen la docencia). La aplicación de los instrumentos se realizó a través del apoyo de los estudiantes de la Escuela Normal Superior José E. Medrano R. durante sus jornadas de observación y práctica en el semestre enero-junio 2018, en turno matutino y vespertino.

\section{Resultados}

Una vez que se aplicaron los instrumentos, se procedió a sistematizar la información. A continuación se muestran los principales hallazgos:

Una de las competencias docentes socioafectivas que se considera fundamental para el establecimiento de un ambiente propicio para el aprendizaje es la empatía. En este tenor, los estudiantes opinan que sus profesores son empáticos en un 49\% en rangos de Casi siempre y Siempre, 
RECIE. Revista Electrónica Científica de Investigación Educativa

Vol. 4, núm. 2, enero-diciembre 2019, pp. 1179-1187.

mientras que un $16 \%$ señala que no lo son en los rangos de Nunca y Casi nunca (Figura 1).

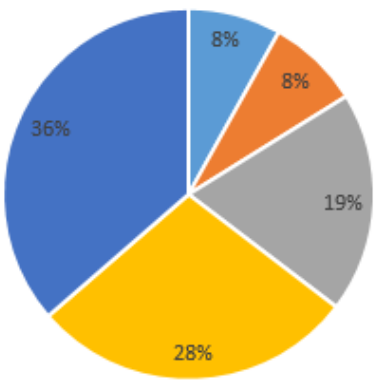

" Nunca "Casi nunca " Regularmente "Casisiempre " Siempre

Figura 1. El docente es empático.

Al cuestionarlos acerca de la confianza que sienten para externar dudas o comentarios, aproximadamente 6 de cada 10 estudiantes indicaron que percibían ese ambiente adecuado para expresar sus inquietudes (Figura 2).

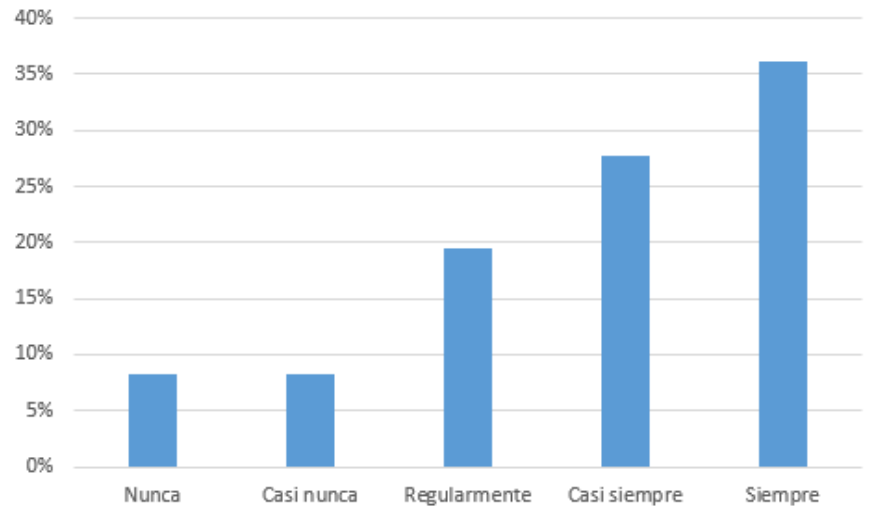

Figura 2. Tengo confianza para externar cualquier duda a mis docentes durante las clases.

Uno de los resultados más significativos en el presente estudio corresponde a la competencia socioafectiva del respeto, pues a diferencia del resto de los hallazgos, se obtuvo una respuesta casi uniforme en todos los estudiantes, al indicar que el $89 \%$ de las ocasiones perciben respeto por parte de sus maestros (Figura 3). 


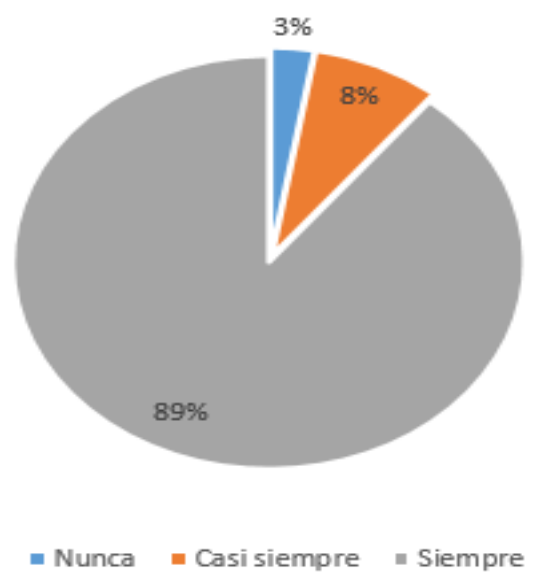

Figura 3. Los profesores brindan respeto.

Finalmente, al cuestionarles acerca del enfoque que los docentes tenían al impartir sus clases, los estudiantes opinaron que aquellos que se enfocan únicamente en contenidos de manera general (Siempre y casi siempre) sólo corresponden al 20\% de los casos; que los profesores que se enfocan en atender asuntos de índole personal con el alumnado representan el 51\% en los mismos rangos y que el $45 \%$ de los profesores combinan ambos aspectos regularmente. Es interesante identificar que los alumnos consideran que existe un $22 \%$ de docentes que Nunca se enfocan únicamente en contenidos, lo cual da la pauta para percibir que además de lo cognitivo, se interesan en el ámbito personal de su alumnado (Tabla 1).

\begin{tabular}{|l|r|r|r|r|r|}
\hline \multicolumn{1}{|c|}{ Se enfocan en: } & \multicolumn{1}{c|}{ Nunca } & Casi nunca & Regularmente & Casi siempre & \multicolumn{1}{c|}{ Siempre } \\
\hline Únicamente en contenidos & $22 \%$ & $19 \%$ & $39 \%$ & $14 \%$ & $6 \%$ \\
\hline Aspectos personales de los estudiantes & $9 \%$ & $18 \%$ & $21 \%$ & $42 \%$ & $9 \%$ \\
\hline Combinan ambos aspectos & $3 \%$ & $12 \%$ & $45 \%$ & $15 \%$ & $24 \%$ \\
\hline
\end{tabular}

Tabla 1. Enfoque de la enseñanza según la perspectiva de los estudiantes.

\section{Discusión}

Desde una perspectiva empírica, parecería que las escuelas secundarias cuentan con profesores que no atienden la emocionalidad de sus estudiantes, sin embargo, después de la aplicación de instrumentos y la sistematización subsecuente, es factible señalar que desde la óptica del alumnado, sus docentes tienden a ser personas empáticas y que primordialmente se conducen con respeto hacia ellos. 
Otro aspecto que permite dar cuenta del tipo de relación que existe entre profesores y estudiantes es la confianza para externar dudas y comentarios durante las clases. Si bien influyen otros aspectos tales como la personalidad del estudiante o las características del grupo, es importante señalar que el profesorado incide también en la generación de una atmósfera en la cual los alumnos perciban la posibilidad de expresar dudas u opiniones en torno a la clase $o$ a situaciones personales.

Un elemento que resulta importante destacar corresponde al enfoque que el profesorado le brinda a su enseñanza, esto es, si durante las sesiones de clase enfatiza los contenidos sin incluir espacio u oportunidad para atender las inquietudes de sus estudiantes, si desayunaron, si se sienten bien, etc. Es interesante observar que los estudiantes consideran que el $22 \%$ de sus profesores nunca se enfocan sólo a los contenidos, lo cual refleja que además de lo conceptual se está considerando la dimensión interpersonal en las clases.

Por tratarse de los primeros hallazgos de un estudio más amplio, es necesario combinar esta información con el resto de las categorías incluidas en los instrumentos, no obstante, es evidente una tendencia del alumnado a describir a sus docentes como personas que atienden lo cognitivo y lo afectivo, la mente y las emociones, sin omitir que en algunos casos, también señalaron que hay docentes que no son empáticos, no generan confianza ni son respetuosos, si bien estos porcentajes son bajos no dejan de ser significativos.

Finalmente, ante la exigencia de un modelo educativo que incluye la dimensión socioemocional en su nueva estructura, surgen muchas incógnitas en torno a la capacitación y/o actualización de los docentes, el desarrollo de competencias socioafectivas que les permitan gestionar sus propias emociones así como intervenir en los estados anímicos de sus grupos y las carencias estructurales de los centros escolares que hacen difícil brindar una atención más cercana (con grupos que en ocasiones rebasan los cuarenta estudiantes en varias escuelas secundarias).

\section{Referencias}

Casassus, J. (2009). La educación del ser emocional. Chile: Índigo/ Cuarto propio

Ducoing, P. y Barrón, C. (2017). La escuela secundaria hoy. Problemas y retos. Revista Mexicana de Investigación Educativa, vol. 22, núm. 72, pp. 930 
Espinosa, M. (2013). La inteligencia emocional del docente de Química del nivel medio superior como factor para el desempeño académico de sus alumnos. México, DF: Universidad Iberoamericana

García, B. (2009). Las dimensiones afectivas de la docencia. Revista Digital Universitaria. 10, (11). Recuperado de http://www.revista.unam.mx/vol.10/num11/art71/art71.pdf

Olson, C. O. y Wyett, J. L. (2000). Teachers need affective competencies. Education, 120. Recuperado de http://findarticles.com/p/articles/ mi_qa3673/ is_200007/ai_n8902469 el 16 de octubre de 2012

Reimers, F. (2001). “Educación, exclusión y justicia social en América Latina”, en C. Ornelas (ed.), Investigación y política educativas: ensayos en honor de Pablo Latapí, México: Santillana, pp. 187-230.

Toro, J. (2005). Educar con co-razón. Bilbao: Desclee de Brouwer, S.A.

UNESCO (1994). Declaración Mundial sobre Educación para Todos. París: UNESCO. 2a . Reimpresión.

UNESCO (2000). Informe Final del Foro Mundial sobre Educación para Todos. Francia: UNESCO

\section{Agradecimientos}

Externo mi gratitud a las autoridades académicas de la Escuela Normal Superior José E. Medrano R. por todas las facilidades otorgadas para la realización del presente estudio, sin embargo, mi mayor agradecimiento es para cada uno de los estudiantes normalistas que de manera generosa invirtieron tiempo y esfuerzo para la aplicación de los instrumentos. Muchas gracias. 
RECIE. Revista Electrónica Científica de Investigación Educativa Vol. 4, núm. 2, enero-diciembre 2019, pp. 1179-1187. 